\title{
Regularity criterion for a weak solution to the three-dimensional magneto-micropolar fluid equations
}

\section{Yinxia Wang*}

\section{"Correspondence:}

yinxia117@126.com

School of Mathematics and

Information Sciences, North China

University of Water Resources and

Electric Power, Zhengzhou, 450011

China

\begin{abstract}
In this paper, a regularity criterion for the 3D magneto-micropolar fluid equations is investigated. A sufficient condition on the derivative of the velocity field in one direction is obtained. More precisely, we prove that if $u_{x_{3}}$ belongs to $L^{\beta}\left(0, T ; L^{\alpha}\left(\mathbb{R}^{3}\right)\right)$ with $\frac{3}{\alpha}+\frac{2}{\beta} \leq 1$ and $\alpha \geq 3$, then the solution $(u, v, b)$ is regular.

MSC: $35 \mathrm{~K} 15 ; 35 \mathrm{~K} 45$
\end{abstract}

Keywords: magneto-micropolar fluid equations; weak solution; regularity criterion

\section{Introduction}

In the paper we investigate the initial value problem for magneto-micropolar fluid equations in $\mathbb{R}^{3}$

$$
\left\{\begin{array}{l}
\partial_{t} u-(\mu+\chi) \Delta u+u \cdot \nabla u-b \cdot \nabla b+\nabla\left(p+\frac{1}{2}|b|^{2}\right)-\chi \nabla \times v=0 \\
\partial_{t} v-\gamma \Delta v-\kappa \nabla \nabla \cdot v+2 \chi v+u \cdot \nabla v-\chi \nabla \times u=0 \\
\partial_{t} b-v \Delta b+u \cdot \nabla b-b \cdot \nabla u=0 \\
\nabla \cdot u=0, \quad \nabla \cdot b=0
\end{array}\right.
$$

with the initial value

$$
t=0: \quad u=u_{0}(x), \quad v=v_{0}(x), \quad b=b_{0}(x)
$$

where $u(t, x), v(t, x), b(t, x)$ and $p(t, x)$ denote the velocity of the fluid, the micro-rotational velocity, magnetic field and hydrostatic pressure, respectively. $\mu$ is the kinematic viscosity, $\chi$ is the vortex viscosity, $\gamma$ and $\kappa$ are spin viscosities and $\frac{1}{v}$ is the magnetic Reynold.

The incompressible magneto-micropolar fluid equations (1.1) have been studied extensively (see [1-6] and [7-10]). The existence and uniqueness of local strong solutions is proved by the Galerkin method in [5]. In [4], the author proved global existence of a strong solution with the small initial data. The existence of weak solutions and the uniqueness of weak solutions in 2D case were established in [6]. Yuan [8] obtained a Beale-Kato-Majda type blow-up criterion for a smooth solution $(u, v, b)$ to the Cauchy problem for (1.1) that

\section{Springer}

(c) 2013 Wang; licensee Springer. This is an Open Access article distributed under the terms of the Creative Commons Attribution License (http://creativecommons.org/licenses/by/2.0), which permits unrestricted use, distribution, and reproduction in any medium, provided the original work is properly cited. 
relies on the vorticity of velocity $\nabla \times u$ only. Wang et al. [10] established a Beale-KatoMajda blow-up criterion of smooth solutions to the 3D magneto-micropolar fluid equation with partial viscosity. Fundamental mathematical issues such as the regularity of weak solutions have generated extensive research and many interesting results have been established (see [1,7] and [9]).

If $b=0,(1.1)$ reduces to micropolar fluid equations. The micropolar fluid equations were first proposed by Eringen [11] (see also [12]). The existence of weak and strong solutions for micropolar fluid equations was obtained by Galdi and Rionero [13] and Yamaguchi [14], respectively. Dong and Chen [15] established regularity criteria of weak solutions to the three-dimensional micropolar fluid equations. In [3], the authors gave sufficient conditions on the kinematics pressure in order to obtain the regularity and uniqueness of weak solutions to the micropolar fluid equations. For more details on regularity criteria, see $[16,17]$ and $[18]$.

If both $v=0$ and $\chi=0$, then equations (1.1) reduce to be magneto-hydrodynamic(MHD) equations. Magnetohydrodynamics (MHD), the science of motion of an electrically conducting fluid in the presence of a magnetic field, consists essentially of the interaction between the fluid velocity and the magnetic field (see [19]). Besides their physical applications, the MHD equations are also mathematically significant. The local existence of solutions to the Cauchy problem (1.1), (1.2) in the usual Sobolev spaces $H^{s}\left(\mathbb{R}^{3}\right)$ was established in [20] for any given initial data $u_{0}, B_{0} \in H^{s}\left(\mathbb{R}^{3}\right), s \geq 3$. But whether the local solution can be extended to a global solution is a challenging open problem in the mathematical fluid mechanics. There are numerous important progresses on the fundamental issue of the regularity for the weak solution to (1.1), (1.2) (see [21-28] and [29-32]).

The purpose of this paper is to establish the regularity criteria of weak solutions to (1.1), (1.2) via the derivative of the velocity in one direction. It is proved that if $\int_{0}^{T}\left\|u_{x_{3}}\right\|_{L^{\alpha}}^{\beta} d t<\infty$ with $\frac{3}{\alpha}+\frac{2}{\beta} \leq 1$ and $\alpha \geq 3$, then the solution $(u, v, b)$ can be extended smoothly beyond $t=T$.

The paper is organized as follows. We first state some important inequalities in Section 2. Then we give the definition of a weak solution and state main results in Section 3, and then we prove the main result in Section 4.

\section{Preliminaries}

In order to prove our main result, we need the following lemma, which may be found in [33] (see also [21, 34] and [35]).

Lemma 2.1 Assume that $\theta, \lambda, \vartheta \in \mathbb{R}$ and satisfy

$$
1 \leq \theta, \quad \lambda<\infty, \quad \frac{1}{\theta}+\frac{2}{\lambda}>1, \quad 1+\frac{3}{\vartheta}=\frac{1}{\theta}+\frac{2}{\lambda} .
$$

Assume that $f \in H^{1}\left(\mathbb{R}^{3}\right), f_{x_{1}}, f_{x_{2}} \in L^{\lambda}\left(\mathbb{R}^{3}\right)$ and $f_{x_{3}} \in L^{\theta}\left(\mathbb{R}^{3}\right)$. Then there exists a positive constant such that

$$
\|f\|_{L^{\vartheta}} \leq C\left\|f_{x_{1}}\right\|_{L^{\lambda}}^{\frac{1}{3}}\left\|f_{x_{2}}\right\|_{L^{\lambda}}^{\frac{1}{3}}\left\|f_{x_{3}}\right\|_{L^{\theta}}^{\frac{1}{3}}
$$


Especially, when $\lambda=2$, there exists a positive constant $C=C(\theta)$ such that

$$
\|f\|_{L^{3 \theta}} \leq C\left\|f_{x_{1}}\right\|_{L^{2}}^{\frac{1}{3}}\left\|f_{x_{2}}\right\|_{L^{2}}^{\frac{1}{3}}\left\|f_{x_{3}}\right\|_{L^{\theta}}^{\frac{1}{3}}
$$

which holds for any $f \in H^{1}\left(\mathbb{R}^{3}\right)$ and $f_{x_{3}} \in L^{\theta}\left(\mathbb{R}^{3}\right)$ with $1 \leq \mu<\infty$.

Lemma 2.2 Let $2 \leq q \leq 6$ and assume that $f \in H^{1}\left(\mathbb{R}^{3}\right)$. Then there exists a positive constant $C=C(q)$ such that

$$
\|f\|_{L^{q}} \leq C\|f\|_{L^{2}}^{\frac{6-q}{2 q}}\left\|\partial_{x_{1}} f\right\|_{L^{2}}^{\frac{q-2}{2 q}}\left\|\partial_{x_{2}} f\right\|_{L^{2}}^{\frac{q-2}{2 q}}\left\|\partial_{x_{3}} f\right\|_{L^{2}}^{\frac{q-2}{2 q}} .
$$

Proof It follows from the interpolating inequality that

$$
\|f\|_{L^{q}} \leq C\|f\|_{L^{2}}^{\frac{6-q}{2 q}}\|f\|_{L^{6}}^{\frac{3 q-6}{2 q}} .
$$

Using (2.2) with $\theta=2$, we obtain

$$
\|f\|_{L^{6}} \leq C\left\|\partial_{x_{1}} f\right\|_{L^{2}}^{\frac{1}{3}}\left\|\partial_{x_{2}} f\right\|_{L^{2}}^{\frac{1}{3}}\left\|\partial_{x_{3}} f\right\|_{L^{2}}^{\frac{1}{3}}
$$

Combining (2.4) and (2.5) immediately yields (2.3).

\section{Main results}

Before stating our main results, we introduce some function spaces. Let

$$
C_{0, \sigma}^{\infty}\left(\mathbb{R}^{3}\right)=\left\{\varphi \in\left(C^{\infty}\left(\mathbb{R}^{3}\right)\right)^{3}: \nabla \cdot \varphi=0\right\} \subset\left(C^{\infty}\left(\mathbb{R}^{3}\right)\right)^{3} .
$$

The subspace

$$
L_{\sigma}^{2}=\overline{C_{0, \sigma}^{\infty}\left(\mathbb{R}^{3}\right)}\|\cdot\|_{L^{2}}=\left\{\varphi \in L^{2}\left(\mathbb{R}^{3}\right): \nabla \cdot \varphi=0\right\}
$$

is obtained as the closure of $C_{0, \sigma}^{\infty}$ with respect to $L^{2}$-norm $\|\cdot\|_{L^{2}} \cdot H_{\sigma}^{r}$ is the closure of $C_{0, \sigma}^{\infty}$ with respect to the $H^{r}$-norm

$$
\|\varphi\|_{H^{r}}=\left\|(I-\Delta)^{\frac{r}{2}} \varphi\right\|_{L^{2}}, \quad r \geq 0 .
$$

Before stating our main results, we give the definition of a weak solution to (1.1), (1.2) (see $[1,7]$ and $[9])$.

Definition 3.1 (Weak solutions) Let $T>0, u_{0}, b_{0} \in L_{\sigma}^{2}\left(\mathbb{R}^{3}\right), v_{0} \in L^{2}\left(\mathbb{R}^{3}\right)$. A measurable $\mathbb{R}^{3}$-valued triple $(u, v, b)$ is said to be a weak solution to $(1.1),(1.2)$ on $[0, T]$ if the following conditions hold:

1.

$$
(u, b) \in L^{\infty}\left(0, T ; L_{\sigma}^{2}\left(\mathbb{R}^{3}\right)\right) \cap L^{2}\left(0, T ; H_{\sigma}^{1}\left(\mathbb{R}^{3}\right)\right)
$$


and

$$
v \in L^{\infty}\left(0, T ; L^{2}\left(\mathbb{R}^{3}\right)\right) \cap L^{2}\left(0, T ; H^{1}\left(\mathbb{R}^{3}\right)\right)
$$

2. (1.1), (1.2) is satisfied in the sense of distributions, i.e., for every $(\varphi, \psi) \in H^{1}\left((0, T) ; H_{\sigma}^{1}\right)$ and $\phi \in H^{1}\left((0, T) ; H^{1}\right)$ with $\varphi(T)=\psi(T)=\phi(T)=0$, the following hold:

$$
\begin{aligned}
& \int_{0}^{T}\left\{-\left\langle u, \partial_{\tau} \varphi\right\rangle+\langle u \cdot \nabla u, \varphi\rangle+(\mu+\chi)\langle\nabla u, \nabla \varphi\rangle\right\} d \tau \\
& \quad-\int_{0}^{T}\{\langle b \cdot \nabla b, \varphi\rangle+\chi\langle\nabla \times v, \varphi\rangle\} d \tau=\left\langle u_{0}, \varphi(0)\right\rangle, \\
& \int_{0}^{T}\left\{-\left\langle v, \partial_{\tau} \phi\right\rangle\right\}+\gamma\langle\nabla v, \nabla \phi\rangle+\kappa\langle\nabla \cdot v, \nabla \phi\rangle+2 \chi\langle v, \phi\rangle d \tau \\
& \quad+\int_{0}^{T}\{\langle u \cdot \nabla v, \phi\rangle-\chi\langle\nabla \times u, \phi\rangle\} d \tau=\left\langle v_{0}, \phi(0)\right\rangle
\end{aligned}
$$

and

$$
\int_{0}^{T}\left\{-\left\langle b, \partial_{\tau} \psi\right\rangle+v\langle\nabla b, \nabla \psi\rangle+\langle u \cdot \nabla b, \psi\rangle-\langle b \cdot \nabla u, \psi\rangle\right\} d \tau=\left\langle b_{0}, \psi(0)\right\rangle .
$$

3. The energy inequality, that is,

$$
\begin{aligned}
& \|u(t)\|_{L^{2}}^{2}+\|v(t)\|_{L^{2}}^{2}+\|b(t)\|_{L^{2}}^{2}+2 \int_{0}^{t}\left(\mu\|\nabla u(\tau)\|_{L^{2}}^{2}+\gamma\|\nabla v(\tau)\|_{L^{2}}^{2}\right) d \tau \\
& \quad+2 \int_{0}^{t}\left(\kappa\|\nabla \cdot v(\tau)\|_{L^{2}}^{2}+\chi\|v(\tau)\|_{L^{2}}^{2}+v\|\nabla b(\tau)\|_{L^{2}}^{2}\right) d \tau \\
& \leq\left\|u_{0}\right\|_{L^{2}}^{2}+\left\|v_{0}\right\|_{L^{2}}^{2}+\left\|b_{0}\right\|_{L^{2}}^{2} .
\end{aligned}
$$

Theorem 3.1 Let $u_{0}, b_{0} \in H_{\sigma}^{1}\left(\mathbb{R}^{3}\right)$ with $v_{0} \in H^{1}\left(\mathbb{R}^{3}\right)$. Assume that $(u, v, b)$ is a weak solution to (1.1), (1.2) on some interval $[0, T]$. If

$$
\Phi(T) \equiv \int_{0}^{T}\left\|u_{x_{3}}\right\|_{L^{\alpha}}^{\beta} d t<\infty
$$

where

$$
\frac{3}{\alpha}+\frac{2}{\beta} \leq 1, \quad \alpha \geq 3
$$

then the solution $(u, v, b)$ can be extended smoothly beyond $t=T$.

\section{Proof of Theorem 3.1}

Proof Multiplying the first equation of (1.1) by $u$ and integrating with respect to $x$ on $\mathbb{R}^{3}$, using integration by parts, we obtain

$$
\frac{1}{2} \frac{d}{d t}\|u(t)\|_{L^{2}}^{2}+(\mu+\chi)\|\nabla u(t)\|_{L^{2}}^{2}=\int_{\mathbb{R}^{3}} b \cdot \nabla b \cdot u d x+\chi \int_{\mathbb{R}^{3}}(\nabla \times v) \cdot u d x .
$$


Similarly, we get

$$
\frac{1}{2} \frac{d}{d t}\|v(t)\|_{L^{2}}^{2}+\gamma\|\nabla v(t)\|_{L^{2}}^{2}+\kappa\|\nabla \cdot v\|_{L^{2}}^{2}+2 \chi\|v\|_{L^{2}}^{2}=\chi \int_{\mathbb{R}^{3}}(\nabla \times u) \cdot v d x
$$

and

$$
\frac{1}{2} \frac{d}{d t}\|b(t)\|_{L^{2}}^{2}+v\|\nabla b(t)\|_{L^{2}}^{2}=\int_{\mathbb{R}^{3}} b \cdot \nabla u \cdot b d x .
$$

Summing up (4.1)-(4.3), we deduce that

$$
\begin{aligned}
& \frac{1 \frac{d}{2}}{d t}\left(\|u(t)\|_{L^{2}}^{2}+\|v(t)\|_{L^{2}}^{2}+\|b(t)\|_{L^{2}}^{2}\right)+(\mu+\chi)\|\nabla u(t)\|_{L^{2}}^{2} \\
& \quad+\gamma\|\nabla v(t)\|_{L^{2}}^{2}+\kappa\|\nabla \cdot v\|_{L^{2}}^{2}+2 \chi\|v\|_{L^{2}}^{2}+v\|\nabla b(t)\|_{L^{2}}^{2} \\
& =\int_{\mathbb{R}^{3}} b \cdot \nabla b \cdot u d x+\chi \int_{\mathbb{R}^{3}}(\nabla \times v) \cdot u d x \\
& \quad+\chi \int_{\mathbb{R}^{3}}(\nabla \times u) \cdot v d x+\int_{\mathbb{R}^{3}} b \cdot \nabla u \cdot b d x .
\end{aligned}
$$

By integration by parts and the Cauchy inequality, we obtain

$$
\chi \int_{\mathbb{R}^{3}}(\nabla \times v) \cdot u d x+\chi \int_{\mathbb{R}^{3}}(\nabla \times u) \cdot v d x \leq \chi\|\nabla u\|_{L^{2}}^{2}+\chi\|v\|_{L^{2}}^{2} .
$$

Using integration by parts, we obtain

$$
\int_{\mathbb{R}^{3}} b \cdot \nabla b \cdot u d x+\int_{\mathbb{R}^{3}} b \cdot \nabla u \cdot b d x=0
$$

Combining (4.4)-(4.6) yields

$$
\begin{aligned}
& \frac{1}{2} \frac{d}{d t}\left(\|u(t)\|_{L^{2}}^{2}+\|v(t)\|_{L^{2}}^{2}+\|b(t)\|_{L^{2}}^{2}\right)+\mu\|\nabla u(t)\|_{L^{2}}^{2} \\
& \quad+\gamma\|\nabla v(t)\|_{L^{2}}^{2}+\kappa\|\nabla \cdot v\|_{L^{2}}^{2}+\chi\|v(t)\|_{L^{2}}^{2}+v\|\nabla b(t)\|_{L^{2}}^{2} \leq 0 .
\end{aligned}
$$

Integrating with respect to $t$, we have

$$
\begin{aligned}
& \|u(t)\|_{L^{2}}^{2}+\|v(t)\|_{L^{2}}^{2}+\|b(t)\|_{L^{2}}^{2}+2 \int_{0}^{t}\left(\mu\|\nabla u(\tau)\|_{L^{2}}^{2}+\gamma\|\nabla v(\tau)\|_{L^{2}}^{2}\right) d \tau \\
& \quad+2 \int_{0}^{t}\left(\kappa\|\nabla \cdot v(\tau)\|_{L^{2}}^{2}+\chi\|v(\tau)\|_{L^{2}}^{2}+v\|\nabla b(\tau)\|_{L^{2}}^{2}\right) d \tau \\
& \leq\left\|u_{0}\right\|_{L^{2}}^{2}+\left\|v_{0}\right\|_{L^{2}}^{2}+\left\|b_{0}\right\|_{L^{2}}^{2} .
\end{aligned}
$$

Differentiating (1.1) with respect to $x_{3}$, we obtain

$$
\left\{\begin{array}{l}
\partial_{t} u_{x_{3}}-(\mu+\chi) \Delta u_{x_{3}}+u_{x_{3}} \cdot \nabla u+u \cdot \nabla u_{x_{3}}-b_{x_{3}} \cdot \nabla b-b \cdot \nabla b_{x_{3}} \\
\quad+\nabla\left(p+\frac{1}{2}|b|^{2}\right)_{x_{3}}-\chi \nabla \times v_{x_{3}}=0, \\
\quad \partial_{t} v_{x_{3}}-\gamma \Delta v_{x_{3}}-\kappa \nabla \cdot \nabla v_{x_{3}}+2 \chi v_{x_{3}}+u_{x_{3}} \cdot \nabla v+u \cdot \nabla v_{x_{3}}-\chi \nabla \times u_{x_{3}}=0, \\
\partial_{t} b_{x_{3}}-v \Delta b_{x_{3}}+u_{x_{3}} \cdot \nabla b+u \cdot \nabla b_{x_{3}}-b_{x_{3}} \cdot \nabla u-b \cdot \nabla u_{x_{3}}=0 .
\end{array}\right.
$$


Taking the inner product of $u_{x_{3}}$ with the first equation of (4.8) and using integration by parts yield

$$
\begin{aligned}
\frac{1}{2} \frac{d}{d t}\left\|u_{x_{3}}(t)\right\|_{L^{2}}^{2}+(\mu+\chi)\left\|\nabla u_{x_{3}}(t)\right\|_{L^{2}}^{2} & \\
= & -\int_{\mathbb{R}^{3}} u_{x_{3}} \cdot \nabla u \cdot u_{x_{3}} d x+\int_{\mathbb{R}^{3}} b_{x_{3}} \cdot \nabla b \cdot u_{x_{3}} d x \\
& \quad+\int_{\mathbb{R}^{3}} b \cdot \nabla b_{x_{3}} \cdot u_{x_{3}} d x+\chi \int_{\mathbb{R}^{3}}\left(\nabla \times v_{x_{3}}\right) \cdot u_{x_{3}} d x .
\end{aligned}
$$

Similarly, we get

$$
\begin{aligned}
& \frac{1}{2} \frac{d}{d t}\left\|v_{x_{3}}(t)\right\|_{L^{2}}^{2}+\gamma\left\|\nabla v_{x_{3}}(t)\right\|_{L^{2}}^{2}+\kappa\left\|\nabla \cdot v_{x_{3}}\right\|_{L^{2}}^{2}+2 \chi\left\|v_{x_{3}}\right\|_{L^{2}}^{2} \\
& \quad=-\int_{\mathbb{R}^{3}} u_{x_{3}} \cdot \nabla v \cdot v_{x_{3}} d x+\chi \int_{\mathbb{R}^{3}}\left(\nabla \times u_{x_{3}}\right) \cdot v_{x_{3}} d x
\end{aligned}
$$

and

$$
\begin{aligned}
& \frac{1}{2} \frac{d}{d t}\left\|b_{x_{3}}(t)\right\|_{L^{2}}^{2}+v\left\|\nabla b_{x_{3}}(t)\right\|_{L^{2}}^{2} \\
& \quad=-\int_{\mathbb{R}^{3}} u_{x_{3}} \cdot \nabla b \cdot b_{x_{3}} d x+\int_{\mathbb{R}^{3}} b_{x_{3}} \cdot \nabla u \cdot b_{x_{3}} d x+\int_{\mathbb{R}^{3}} b \cdot \nabla u_{x_{3}} \cdot b_{x_{3}} d x .
\end{aligned}
$$

Combining (4.9)-(4.11) yields

$$
\begin{aligned}
\frac{1}{2} \frac{d}{d t} & \left(\left\|u_{x_{3}}(t)\right\|_{L^{2}}^{2}+\left\|v_{x_{3}}(t)\right\|_{L^{2}}^{2}+\left\|b_{x_{3}}(t)\right\|_{L^{2}}^{2}\right)+(\mu+\chi)\left\|\nabla u_{x_{3}}(t)\right\|_{L^{2}}^{2} \\
& +\gamma\left\|\nabla v_{x_{3}}(t)\right\|_{L^{2}}^{2}+\kappa\left\|\nabla \cdot v_{x_{3}}\right\|_{L^{2}}^{2}+2 \chi\left\|v_{x_{3}}\right\|_{L^{2}}^{2}+v\left\|\nabla b_{x_{3}}(t)\right\|_{L^{2}}^{2} \\
= & -\int_{\mathbb{R}^{3}} u_{x_{3}} \cdot \nabla u \cdot u_{x_{3}} d x+\int_{\mathbb{R}^{3}} b_{x_{3}} \cdot \nabla b \cdot u_{x_{3}} d x+\int_{\mathbb{R}^{3}} b \cdot \nabla b_{x_{3}} \cdot u_{x_{3}} d x \\
& +\chi \int_{\mathbb{R}^{3}}\left(\nabla \times v_{x_{3}}\right) \cdot u_{x_{3}} d x-\int_{\mathbb{R}^{3}} u_{x_{3}} \cdot \nabla v \cdot v_{x_{3}} d x+\chi \int_{\mathbb{R}^{3}}\left(\nabla \times u_{x_{3}}\right) \cdot v_{x_{3}} d x \\
& -\int_{\mathbb{R}^{3}} u_{x_{3}} \cdot \nabla b \cdot b_{x_{3}} d x+\int_{\mathbb{R}^{3}} b_{x_{3}} \cdot \nabla u \cdot b_{x_{3}} d x+\int_{\mathbb{R}^{3}} b \cdot \nabla u_{x_{3}} \cdot b_{x_{3}} d x .
\end{aligned}
$$

Using integration by parts and the Cauchy inequality, we obtain

$$
\chi \int_{\mathbb{R}^{3}}\left(\nabla \times v_{x_{3}}\right) \cdot u_{x_{3}} d x+\chi \int_{\mathbb{R}^{3}}\left(\nabla \times u_{x_{3}}\right) \cdot v_{x_{3}} d x \leq \chi\left\|\nabla u_{x_{3}}\right\|_{L^{2}}^{2}+\chi\left\|v_{x_{3}}\right\|_{L^{2}}^{2} .
$$

Using integration by parts, we have

$$
\int_{\mathbb{R}^{3}} b \cdot \nabla b_{x_{3}} \cdot u_{x_{3}} d x+\int_{\mathbb{R}^{3}} b \cdot \nabla u_{x_{3}} \cdot b_{x_{3}} d x=0 .
$$

Combining (4.12)-(4.14) yields

$$
\begin{aligned}
& \frac{1}{2} \frac{d}{d t}\left(\left\|u_{x_{3}}(t)\right\|_{L^{2}}^{2}+\left\|v_{x_{3}}(t)\right\|_{L^{2}}^{2}+\left\|b_{x_{3}}(t)\right\|_{L^{2}}^{2}\right)+\mu\left\|\nabla u_{x_{3}}(t)\right\|_{L^{2}}^{2} \\
& \quad+\gamma\left\|\nabla v_{x_{3}}(t)\right\|_{L^{2}}^{2}+\kappa\left\|\nabla \cdot v_{x_{3}}\right\|_{L^{2}}^{2}+\chi\left\|v_{x_{3}}(t)\right\|_{L^{2}}^{2}+v\left\|\nabla b_{x_{3}}(t)\right\|_{L^{2}}^{2}
\end{aligned}
$$




$$
\begin{aligned}
\leq & -\int_{\mathbb{R}^{3}} u_{x_{3}} \cdot \nabla u \cdot u_{x_{3}} d x+\int_{\mathbb{R}^{3}} b_{x_{3}} \cdot \nabla b \cdot u_{x_{3}} d x-\int_{\mathbb{R}^{3}} u_{x_{3}} \cdot \nabla v \cdot v_{x_{3}} d x \\
& \quad-\int_{\mathbb{R}^{3}} u_{x_{3}} \cdot \nabla b \cdot b_{x_{3}} d x+\int_{\mathbb{R}^{3}} b_{x_{3}} \cdot \nabla u \cdot b_{x_{3}} d x \\
\triangleq & I_{1}+I_{2}+I_{3}+I_{4}+I_{5} .
\end{aligned}
$$

In what follows, we estimate $I_{j}(j=1,2, \ldots, 5)$. By integration by parts and the Hölder inequality, we obtain

$$
I_{1} \leq C\left\|\nabla u_{x_{3}}\right\|_{L^{2}}\left\|u_{x_{3}}\right\|_{L^{\varrho}}\|u\|_{L^{3 \alpha}}
$$

where

$$
\frac{1}{\varrho}+\frac{1}{3 \alpha}=\frac{1}{2}, \quad 2 \leq \varrho \leq 6
$$

It follows from the interpolating inequality that

$$
\left\|u_{x_{3}}\right\|_{L^{\varrho}} \leq C\left\|u_{x_{3}}\right\|_{L^{2}}^{1-3\left(\frac{1}{2}-\frac{1}{\varrho}\right)}\left\|\nabla u_{x_{3}}\right\|_{L^{2}}^{3\left(\frac{1}{2}-\frac{1}{\varrho}\right)} .
$$

From (2.2), we get

$$
\begin{aligned}
I_{1} & \leq C\left\|\nabla u_{x_{3}}\right\|_{L^{2}}\left\|u_{x_{3}}\right\|_{L^{2}}^{1-3\left(\frac{1}{2}-\frac{1}{\varrho}\right)}\left\|\nabla u_{x_{3}}\right\|_{L^{2}}^{3\left(\frac{1}{2}-\frac{1}{\varrho}\right)}\|\nabla u\|_{L^{2}}^{\frac{2}{3}}\left\|u_{x_{3}}\right\|_{L^{\alpha}}^{\frac{1}{3}} \\
& \leq C\left\|\nabla u_{x_{3}}\right\|_{L^{2}}^{1+3\left(\frac{1}{2}-\frac{1}{\varrho}\right)}\left\|u_{x_{3}}\right\|_{L^{2}}^{1-3\left(\frac{1}{2}-\frac{1}{\varrho}\right)}\|\nabla u\|_{L^{2}}^{\frac{2}{3}}\left\|u_{x_{3}}\right\|_{L^{\alpha}}^{\frac{1}{3}} \\
& \leq \frac{\mu}{2}\left\|\nabla u_{x_{3}}\right\|_{L^{2}}^{2}+C\left\|u_{x_{3}}\right\|_{L^{2}}^{2}\|\nabla u\|_{L^{2}}^{2 q}\left\|u_{x_{3}}\right\|_{L^{\alpha}}^{q}
\end{aligned}
$$

where

$$
q=\frac{2}{3-9\left(\frac{1}{2}-\frac{1}{\varrho}\right)}=\frac{2}{3\left(1-\frac{1}{\alpha}\right)} .
$$

When $\alpha \geq 3$, we have $2 q \leq 2$, and the application of the Young inequality yields

$$
I_{1} \leq \frac{\mu}{2}\left\|\nabla u_{x_{3}}\right\|_{L^{2}}^{2}+C\left\|u_{x_{3}}\right\|_{L^{2}}^{2}\left(\|\nabla u\|_{L^{2}}^{2}+\left\|u_{x_{3}}\right\|_{L^{\alpha}}^{\delta}\right),
$$

where

$$
\frac{3}{\alpha}+\frac{2}{\delta}=1
$$

From integration by parts and the Hölder inequality, we obtain

$$
\begin{aligned}
I_{2} & \leq C\|\nabla b\|_{L^{2}}\left\|b_{x_{3}}\right\|_{L^{\frac{2 \alpha}{\alpha-2}}}\left\|u_{x_{3}}\right\|_{L^{\alpha}} \leq C\|\nabla b\|_{L^{2}}\left\|u_{x_{3}}\right\|_{L^{\alpha}}\left\|b_{x_{3}}\right\|_{L^{2}}^{1-\frac{3}{\alpha}}\left\|\nabla b_{x_{3}}\right\|_{L^{2}}^{\frac{3}{\alpha}} \\
& \leq \frac{v}{6}\left\|\nabla b_{x_{3}}\right\|_{L^{2}}^{2}+\|\nabla b\|_{L^{2}}^{\frac{2 \alpha}{2 \alpha-3}}\left\|u_{x_{3}}\right\|_{L^{\alpha}}^{\frac{2 \alpha}{2 \alpha-3}}\left\|b_{x_{3}}\right\|_{L^{2}}^{\frac{2 \alpha-6}{2 \alpha-3}} \\
& \leq \frac{v}{6}\left\|\nabla b_{x_{3}}\right\|_{L^{2}}^{2}+C\left(\|\nabla b\|_{L^{2}}^{2}+\left\|u_{x_{3}}\right\|_{L^{\alpha}}^{\delta}\right)\left\|b_{x_{3}}\right\|_{L^{2}}^{\frac{2 \alpha-6}{2 \alpha-3}}
\end{aligned}
$$


where

$$
\frac{3}{\alpha}+\frac{2}{\delta}=1
$$

Similarly,

$$
\begin{aligned}
I_{3} & \leq C\|\nabla v\|_{L^{2}}\left\|v_{x_{3}}\right\|_{L^{\frac{2 \alpha}{\alpha-2}}}\left\|u_{x_{3}}\right\|_{L^{\alpha}} \\
& \leq C\|\nabla v\|_{L^{2}}\left\|u_{x_{3}}\right\|_{L^{\alpha}}\left\|v_{x_{3}}\right\|_{L^{2}}^{1-\frac{3}{\alpha}}\left\|\nabla v_{x_{3}}\right\|_{L^{2}}^{\frac{3}{\alpha}} \\
& \leq \frac{\gamma}{2}\left\|\nabla v_{x_{3}}\right\|_{L^{2}}^{2}+\|\nabla v\|_{L^{2}}^{\frac{2 \alpha}{2 \alpha-3}}\left\|u_{x_{3}}\right\|_{L^{\alpha}}^{\frac{2 \alpha-3}{2 \alpha-3}}\left\|v_{x_{3}}\right\|_{L^{2}}^{\frac{2 \alpha-6}{2 \alpha-3}} \\
& \leq \frac{\gamma}{2}\left\|\nabla v_{x_{3}}\right\|_{L^{2}}^{2}+C\left(\|\nabla v\|_{L^{2}}^{2}+\left\|u_{x_{3}}\right\|_{L^{\alpha}}^{\delta}\right)\left\|v_{x_{3}}\right\|_{L^{2}}^{\frac{2 \alpha-3}{2 \alpha-3}},
\end{aligned}
$$

and

$$
\begin{aligned}
I_{4} & \leq C\|\nabla b\|_{L^{2}}\left\|b_{x_{3}}\right\|_{L^{\frac{2 \alpha}{\alpha-2}}}\left\|u_{x_{3}}\right\|_{L^{\alpha}} \\
& \leq C\|\nabla b\|_{L^{2}}\left\|u_{x_{3}}\right\|_{L^{\alpha}}\left\|b_{x_{3}}\right\|_{L^{2}}^{1-\frac{3}{\alpha}}\left\|\nabla b_{x_{3}}\right\|_{L^{2}}^{\frac{3}{\alpha}} \\
& \leq \frac{v}{6}\left\|\nabla b_{x_{3}}\right\|_{L^{2}}^{2}+\|\nabla b\|_{L^{2}}^{\frac{2 \alpha}{2 \alpha-3}}\left\|u_{x_{3}}\right\|_{L^{\alpha}}^{\frac{2 \alpha}{2 \alpha-3}}\left\|b_{x_{3}}\right\|_{L^{2}}^{\frac{2 \alpha-6}{2 \alpha-3}} \\
& \leq \frac{v}{6}\left\|\nabla b_{x_{3}}\right\|_{L^{2}}^{2}+C\left(\|\nabla b\|_{L^{2}}^{2}+\left\|u_{x_{3}}\right\|_{L^{\alpha}}^{\delta}\right)\left\|b_{x_{3}}\right\|_{L^{2}}^{\frac{2 \alpha-6}{2 \alpha-3}}
\end{aligned}
$$

where

$$
\frac{3}{\alpha}+\frac{2}{\delta}=1
$$

By integration by parts and the inequality, we have

$$
\begin{aligned}
I_{5} & \leq C\left\|\nabla b_{x_{3}}\right\|_{L^{2}}\left\|b_{x_{3}}\right\|_{L^{\sigma}}\|u\|_{L^{3 \alpha}} \\
& \leq C\left\|\nabla b_{x_{3}}\right\|_{L^{2}}\left\|b_{x_{3}}\right\|_{L^{2}}^{1-3\left(\frac{1}{2}-\frac{1}{\sigma}\right)}\left\|\nabla b_{x_{3}}\right\|_{L^{2}}^{3\left(\frac{1}{2}-\frac{1}{\sigma}\right)}\|\nabla u\|_{L^{2}}^{2}\left\|u_{x_{3}}\right\|_{L^{2}}^{\frac{1}{3}} \\
& \leq \frac{v}{6}\left\|\nabla b_{x_{3}}\right\|_{L^{2}}^{2}+C\left\|b_{x_{3}}\right\|_{L^{2}}^{2}\|\nabla u\|_{L^{2}}^{2 q}\left\|u_{x_{3}}\right\|_{L^{\alpha}}^{q},
\end{aligned}
$$

where

$$
q=\frac{2}{3\left(1-\frac{1}{\alpha}\right)} .
$$

When $\alpha \geq 3$, we have $2 q \leq 2$, and the application of the Young inequality yields

$$
I_{5} \leq \frac{v}{6}\left\|\nabla b_{x_{3}}\right\|_{L^{2}}^{2}+C\left\|b_{x_{3}}\right\|_{L^{2}}^{2}\left(\|\nabla u\|_{L^{2}}^{2}+\left\|u_{x_{3}}\right\|_{L^{\alpha}}^{\delta}\right),
$$

where

$$
\frac{3}{\alpha}+\frac{2}{\delta}=1
$$


Combining (4.15)-(4.20) yields

$$
\begin{aligned}
& \frac{d}{d t}\left(\left\|u_{x_{3}}\right\|_{L^{2}}^{2}+\left\|b_{x_{3}}\right\|_{L^{2}}^{2}+\left\|b_{x_{3}}\right\|_{L^{2}}^{2}\right)+\mu\left\|\nabla u_{x_{3}}\right\|_{L^{2}}^{2} \\
& \quad+\gamma\left\|\nabla v_{x_{3}}\right\|_{L^{2}}^{2}+\kappa\left\|\nabla \cdot v_{x_{3}}\right\|_{L^{2}}^{2}+\chi\left\|v_{x_{3}}\right\|_{L^{2}}^{2}+v\left\|\nabla b_{x_{3}}\right\|_{L^{2}}^{2} \\
& \leq C\left(\left\|u_{x_{3}}\right\|_{L^{2}}^{2}+\left\|b_{x_{3}}\right\|_{L^{2}}^{2}\right)\left(\|\nabla u\|_{L^{2}}^{2}+\left\|u_{x_{3}}\right\|_{L^{\alpha}}^{\delta}\right) \\
& \quad+C\left(\|\nabla v\|_{L^{2}}^{2}+\|\nabla b\|_{L^{2}}^{2}+\left\|u_{x_{3}}\right\|_{L^{\alpha}}^{\delta}\right)\left(\left\|v_{x_{3}}\right\|_{L^{2}}^{\frac{2 \alpha-6}{2 \alpha-3}}+\left\|b_{x_{3}}\right\|_{L^{2}}^{\frac{2 \alpha-6}{2 \alpha-3}}\right) .
\end{aligned}
$$

From the Gronwall inequality, we get

$$
\begin{aligned}
& \left\|u_{x_{3}}\right\|_{L^{2}}^{2}+\left\|b_{x_{3}}\right\|_{L^{2}}^{2}+\left\|b_{x_{3}}\right\|_{L^{2}}^{2}+\mu \int_{0}^{t}\left\|\nabla u_{x_{3}}\right\|_{L^{2}}^{2} d \tau \\
& \quad+\int_{0}^{t}\left(\gamma\left\|\nabla v_{x_{3}}\right\|_{L^{2}}^{2}+\kappa\left\|\nabla \cdot v_{x_{3}}\right\|_{L^{2}}^{2}+\chi\left\|v_{x_{3}}\right\|_{L^{2}}^{2}+v\left\|\nabla b_{x_{3}}\right\|_{L^{2}}^{2}\right) d \tau \\
& \leq C e^{\left(\left\|u_{0}\right\|_{L^{2}}^{2}+\left\|v_{0}\right\|_{L^{2}}^{2}+\left\|b_{0}\right\|_{L^{2}}^{2}\right)} e^{\Phi(t)}\left[\left\|u_{0}\right\|_{H^{1}}^{2}+\left\|v_{0}\right\|_{H^{1}}^{2}+\left\|b_{0}\right\|_{H^{1}}^{2}\right. \\
& \left.\quad+C\left(\left\|u_{0}\right\|_{L^{2}}^{2}+\left\|v_{0}\right\|_{L^{2}}^{2}+\left\|b_{0}\right\|_{L^{2}}^{2}+\Phi(t)\right)^{\frac{2 \alpha-3}{\alpha}}\right] .
\end{aligned}
$$

Multiplying the first equation of (1.1) by $-\Delta u$ and integrating with respect to $x$ on $\mathbb{R}^{3}$, and then using integration by parts, we obtain

$$
\begin{aligned}
& \frac{1}{2} \frac{d}{d t}\|\nabla u(t)\|_{L^{2}}^{2}+(\mu+\chi)\|\Delta u\|_{L^{2}}^{2} \\
& \quad=\int_{\mathbb{R}^{3}} u \cdot \nabla u \cdot \Delta u d x-\int_{\mathbb{R}^{3}} b \cdot \nabla b \cdot \Delta u d x-\chi \int_{\mathbb{R}^{3}}(\nabla \times v) \cdot \Delta u d x .
\end{aligned}
$$

Similarly, we get

$$
\begin{aligned}
& \frac{1}{2} \frac{d}{d t}\|\nabla v(t)\|_{L^{2}}^{2}+\gamma\|\Delta v\|_{L^{2}}^{2}+\kappa\|\nabla \nabla \cdot v\|_{L^{2}}^{2}+2 \chi\|\nabla v\|_{L^{2}}^{2} \\
& \quad=\int_{\mathbb{R}^{3}} u \cdot \nabla v \cdot \Delta v d x-\chi \int_{\mathbb{R}^{3}}(\nabla \times u) \cdot \Delta v d x
\end{aligned}
$$

and

$$
\begin{aligned}
& \frac{1}{2} \frac{d}{d t}\|\nabla b(t)\|_{L^{2}}^{2}+v\|\Delta b\|_{L^{2}}^{2} \\
& \quad=\int_{\mathbb{R}^{3}} u \cdot \nabla b \cdot \Delta b d x-\int_{\mathbb{R}^{3}} b \cdot \nabla u \cdot \Delta b d x .
\end{aligned}
$$

Collecting (4.22)-(4.24) yields

$$
\begin{aligned}
& \frac{1}{2} \frac{d}{d t}\left(\|\nabla u(t)\|_{L^{2}}^{2}+\|\nabla v(t)\|_{L^{2}}^{2}+\|\nabla b(t)\|_{L^{2}}^{2}\right)+(\mu+\chi)\|\Delta u\|_{L^{2}}^{2} \\
& \quad+\gamma\|\Delta v\|_{L^{2}}^{2}+\kappa\|\nabla \nabla \cdot v\|_{L^{2}}^{2}+2 \chi\|\nabla v\|_{L^{2}}^{2}+v\|\Delta b\|_{L^{2}}^{2} \\
& =\int_{\mathbb{R}^{3}} u \cdot \nabla u \cdot \Delta u d x-\int_{\mathbb{R}^{3}} b \cdot \nabla b \cdot \Delta u d x-\chi \int_{\mathbb{R}^{3}}(\nabla \times v) \cdot \Delta u d x
\end{aligned}
$$




$$
\begin{aligned}
& +\int_{\mathbb{R}^{3}} u \cdot \nabla v \cdot \Delta v d x-\chi \int_{\mathbb{R}^{3}}(\nabla \times u) \cdot \Delta v d x \\
& +\int_{\mathbb{R}^{3}} u \cdot \nabla b \cdot \Delta b d x-\int_{\mathbb{R}^{3}} b \cdot \nabla u \cdot \Delta b d x .
\end{aligned}
$$

Thanks to integration by parts and the Cauchy inequality, we get

$$
-\chi \int_{\mathbb{R}^{3}}(\nabla \times v) \cdot \Delta u d x-\chi \int_{\mathbb{R}^{3}}(\nabla \times u) \cdot \Delta v d x \leq \chi\|\Delta u\|_{L^{2}}^{2}+\chi\|\nabla v\|_{L^{2}}^{2} .
$$

It follows from (4.25)-(4.26) and integration by parts that

$$
\begin{aligned}
\frac{1}{2} \frac{d}{d t} & \left(\|\nabla u(t)\|_{L^{2}}^{2}+\|\nabla v(t)\|_{L^{2}}^{2}+\|\nabla b(t)\|_{L^{2}}^{2}\right)+\mu\|\Delta u\|_{L^{2}}^{2} \\
& +\gamma\|\Delta v\|_{L^{2}}^{2}+\kappa\|\nabla \nabla \cdot v\|_{L^{2}}^{2}+\chi\|\nabla v\|_{L^{2}}^{2}+v\|\Delta b\|_{L^{2}}^{2} \\
\leq & -\int_{\mathbb{R}^{3}} \nabla u \cdot \nabla u \cdot \nabla u d x+\int_{\mathbb{R}^{3}} \nabla b \cdot \nabla b \cdot \nabla u d x-\int_{\mathbb{R}^{3}} \nabla u \cdot \nabla v \cdot \nabla v d x \\
& \quad-\int_{\mathbb{R}^{3}} \nabla u \cdot \nabla b \cdot \nabla b d x+\int_{\mathbb{R}^{3}} \nabla b \cdot \nabla u \cdot \nabla b d x \\
\triangleq & J_{1}+J_{2}+J_{3}+J_{4}+J_{5} .
\end{aligned}
$$

In what follows, we estimate $J_{i}(i=1, \ldots, 5)$.

By (2.3) and the Young inequality, we deduce that

$$
\begin{aligned}
J_{1} & \leq C\|\nabla u\|_{L^{3}}^{3} \leq C\|\nabla u\|_{L^{2}}^{\frac{3}{2}}\left\|\nabla_{\tilde{x}} \nabla u\right\|_{L^{2}}\left\|\nabla u_{x_{3}}\right\|_{L^{2}}^{\frac{1}{2}} \\
& \leq \frac{\mu}{10}\left\|\nabla_{\tilde{x}} \nabla u\right\|_{L^{2}}^{2}+C\|\nabla u\|_{L^{2}}^{3}\left\|\nabla u_{x_{3}}\right\|_{L^{2}} \\
& \leq \frac{\mu}{10}\left\|\nabla_{\tilde{x}} \nabla u\right\|_{L^{2}}^{2}+C\left(\|\nabla u\|_{L^{2}}^{2}+\left\|\nabla u_{x_{3}}\right\|_{L^{2}}^{2}\right)\|\nabla u\|_{L^{2}}^{2} .
\end{aligned}
$$

By (2.3) and the Young inequality, we have

$$
\begin{aligned}
J_{2} \leq & \|\nabla u\|_{L^{3}}\|\nabla b\|_{L^{3}}^{2} \\
\leq & C\|\nabla u\|_{L^{2}}^{\frac{1}{2}}\left\|\nabla_{\tilde{x}} \nabla u\right\|_{L^{2}}^{\frac{1}{3}}\left\|\nabla u_{x_{3}}\right\|_{L^{2}}^{\frac{1}{6}}\|\nabla b\|_{L^{2}}\left\|\nabla_{\tilde{x}} \nabla b\right\|_{L^{2}}^{\frac{2}{3}}\left\|\nabla b_{x_{3}}\right\|_{L^{2}}^{\frac{1}{3}} \\
\leq & \frac{\mu}{10}\left\|\nabla_{\tilde{x}} \nabla u\right\|_{L^{2}}^{2}+C\|\nabla u\|_{L^{2}}^{\frac{3}{5}}\left\|\nabla u_{x_{3}}\right\|_{L^{2}}^{\frac{1}{5}}\|\nabla b\|_{L^{2}}^{\frac{6}{5}}\left\|\nabla_{\tilde{x}} \nabla b\right\|_{L^{2}}^{\frac{4}{5}}\left\|\nabla b_{x_{3}}\right\|_{L^{2}}^{\frac{2}{5}} \\
\leq & \frac{\mu}{10}\left\|\nabla_{\tilde{x}} \nabla u\right\|_{L^{2}}^{2}+\frac{v}{6}\left\|\nabla_{\tilde{x}} \nabla b\right\|_{L^{2}}^{2}+C\|\nabla u\|_{L^{2}}\left\|\nabla u_{x_{3}}\right\|_{L^{2}}^{\frac{1}{3}}\|\nabla b\|_{L^{2}}^{2}\left\|\nabla b_{x_{3}}\right\|_{L^{2}}^{\frac{2}{3}} \\
\leq & \frac{\mu}{10}\left\|\nabla_{\tilde{x}} \nabla u\right\|_{L^{2}}^{2}+\frac{v}{6}\left\|\nabla_{\tilde{x}} \nabla b\right\|_{L^{2}}^{2} \\
& +C\|\nabla b\|_{L^{2}}^{2}\left(\|\nabla u\|_{L^{2}}^{2}+\left\|\nabla u_{x_{3}}\right\|_{L^{2}}^{2}+\left\|\nabla b_{x_{3}}\right\|_{L^{2}}^{2}\right) .
\end{aligned}
$$

Similarly, we obtain

$$
\begin{aligned}
J_{3} \leq & \|\nabla u\|_{L^{3}}\|\nabla v\|_{L^{3}}^{2} \\
\leq & \frac{\mu}{10}\left\|\nabla_{\tilde{x}} \nabla u\right\|_{L^{2}}^{2}+\frac{\gamma}{2}\left\|\nabla_{\tilde{x}} \nabla v\right\|_{L^{2}}^{2} \\
& +C\|\nabla v\|_{L^{2}}^{2}\left(\|\nabla u\|_{L^{2}}^{2}+\left\|\nabla u_{x_{3}}\right\|_{L^{2}}^{2}+\left\|\nabla v_{x_{3}}\right\|_{L^{2}}^{2}\right),
\end{aligned}
$$




$$
\begin{aligned}
J_{4} \leq & \|\nabla u\|_{L^{3}}\|\nabla b\|_{L^{3}}^{2} \\
\leq & \frac{\mu}{10}\left\|\nabla_{\tilde{x}} \nabla u\right\|_{L^{2}}^{2}+\frac{v}{6}\left\|\nabla_{\tilde{x}} \nabla b\right\|_{L^{2}}^{2} \\
& +C\|\nabla b\|_{L^{2}}^{2}\left(\|\nabla u\|_{L^{2}}^{2}+\left\|\nabla u_{x_{3}}\right\|_{L^{2}}^{2}+\left\|\nabla b_{x_{3}}\right\|_{L^{2}}^{2}\right)
\end{aligned}
$$

and

$$
\begin{aligned}
J_{5} \leq & \|\nabla u\|_{L^{3}}\|\nabla b\|_{L^{3}}^{2} \\
\leq & \frac{\mu}{10}\left\|\nabla_{\tilde{x}} \nabla u\right\|_{L^{2}}^{2}+\frac{v}{6}\left\|\nabla_{\tilde{x}} \nabla b\right\|_{L^{2}}^{2} \\
& +C\|\nabla b\|_{L^{2}}^{2}\left(\|\nabla u\|_{L^{2}}^{2}+\left\|\nabla u_{x_{3}}\right\|_{L^{2}}^{2}+\left\|\nabla b_{x_{3}}\right\|_{L^{2}}^{2}\right) .
\end{aligned}
$$

Combining (4.27)-(4.32) yields

$$
\begin{aligned}
\frac{d}{d t}\left(\|\nabla u(t)\|_{L^{2}}^{2}+\|\nabla v(t)\|_{L^{2}}^{2}+\|\nabla b(t)\|_{L^{2}}^{2}\right)+\mu\|\Delta u\|_{L^{2}}^{2} \\
\quad+\gamma\|\Delta v\|_{L^{2}}^{2}+\kappa\|\nabla \nabla \cdot v\|_{L^{2}}^{2}+\chi\|\nabla v\|_{L^{2}}^{2}+v\|\Delta b\|_{L^{2}}^{2} \\
\leq C\left(\|\nabla u\|_{L^{2}}^{2}+\|\nabla v\|_{L^{2}}^{2}+\|\nabla b\|_{L^{2}}^{2}\right) \\
\quad \times\left(\|\nabla u\|_{L^{2}}^{2}+\left\|\nabla u_{x_{3}}\right\|_{L^{2}}^{2}+\left\|\nabla v_{x_{3}}\right\|_{L^{2}}^{2}+\left\|\nabla b_{x_{3}}\right\|_{L^{2}}^{2}\right) .
\end{aligned}
$$

From (4.33), the Gronwall inequality, (4.7) and (4.21), we know that $(u, v, b) \in L^{\infty}(0, T$; $\left.H^{1}\left(\mathbb{R}^{3}\right)\right)$. Thus, $(u, v, b)$ can be extended smoothly beyond $t=T$. We have completed the proof of Theorem 3.1.

\section{Competing interests}

The author declares that she has no competing interests.

\section{Author's contributions}

The author completed the paper herself. The author read and approved the final manuscript.

Received: 30 January 2013 Accepted: 4 March 2013 Published: 25 March 2013

\section{References}

1. Gala, S: Regularity criteria for the 3D magneto-micropolar fluid equations in the Morrey-Campanato space. Nonlinear Differ. Equ. Appl. 17, 181-194 (2010)

2. Ortega-Torres, EE, Rojas-Medar, MA: On the uniqueness and regularity of the weak solution for magneto-micropolar fluid equations. Rev. Mat. Apl. 17, 75-90 (1996)

3. Ortega-Torres, EE, Rojas-Medar, MA: On the regularity for solutions of the micropolar fluid equations. Rend. Semin. Mat. Univ. Padova 122, 27-37 (2009)

4. Ortega-Torres, EE, Rojas-Medar, MA: Magneto-micropolar fluid motion: global existence of strong solutions. Abstr. Appl. Anal. 4, 109-125 (1999)

5. Rojas-Medar, MA: Magneto-micropolar fluid motion: existence and uniqueness of strong solutions. Math. Nachr. 188, 301-319 (1997)

6. Rojas-Medar, MA, Boldrini, JL: Magneto-micropolar fluid motion: existence of weak solutions. Rev. Mat. Complut. 11, 443-460 (1998)

7. Yuan, B: Regularity of weak solutions to magneto-micropolar fluid equations. Acta Math. Sci. 30, 1469-1480 (2010)

8. Yuan, J: Existence theorem and blow-up criterion of the strong solutions to the magneto-micropolar fluid equations. Math. Methods Appl. Sci. 31, 1113-1130 (2008)

9. Zhang, Z, Yao, A, Wang, $X: A$ regularity criterion for the 3D magneto-micropolar fluid equations in Triebel-Lizorkin spaces. Nonlinear Anal. 74, 2220-2225 (2011)

10. Wang, Y, Hu, L, Wang, Y: A Beale-Kato-Madja criterion for magneto-micropolar fluid equations with partial viscosity. Bound. Value Probl. 2011, Article ID 128614 (2011)

11. Eringen, AC: Theory of micropolar fluids. J. Math. Mech. 16, 1-18 (1966)

12. Lukaszewicz, G: Micropolar Fluids: Theory and Applications. Modeling and Simulation in Science, Engineering and Technology. Birkhäuser, Boston (1999) 
13. Galdi, GP, Rionero, S: A note on the existence and uniqueness of solutions of the micropolar fluid equations. Int. J. Eng. Sci. 15, 105-108 (1977)

14. Yamaguchi, N: Existence of global strong solution to the micropolar fluid system in a bounded domain. Math. Methods Appl. Sci. 28, 1507-1526 (2005)

15. Dong, B, Chen, Z: Regularity criteria of weak solutions to the three-dimensional micropolar flows. J. Math. Phys. 50, Article ID 103525 (2009)

16. Wang, Y, Chen, Z: Regularity criterion for weak solution to the 3D micropolar fluid equations. J. Appl. Math. 2011, Article ID 456547 (2011)

17. Dong, B, Jia, Y, Chen, Z: Pressure regularity criteria of the three-dimensional micropolar fluid flows. Math. Methods Appl. Sci. 34, 595-606 (2011)

18. Wang, Y, Yuan, H: A logarithmically improved blow-up criterion for smooth solutions to the 3D micropolar fluid equations. Nonlinear Anal., Real World Appl. 13, 1904-1912 (2012)

19. Lifschitz, AE: Magnetohydrodynamics and spectral theory. In: Developments in Electromagnetic Theory and Applications, vol. 4. Kluwer Academic, Dordrecht (1989)

20. Sermange, M, Temam, R: Some mathematical questions related to the MHD equations. Commun. Pure Appl. Math. 36, 635-666 (1983)

21. Cao, C, Wu, J: Two regularity criteria for the 3D equations. J. Differ. Equ. 248, 2263-2274 (2010)

22. Gala, S: Extension criterion on regularity for weak solutions to the 3D MHD equations. Math. Methods Appl. Sci. 33, 1496-1503 (2010)

23. He, C, Xin, Z: On the regularity of solutions to the magnetohydrodynamic equations. J. Differ. Equ. 213, 235-254 (2005)

24. He, C, Wang, Y: On the regularity for weak solutions to the magnetohydrodynamic equations. J. Differ. Equ. 238, 1-17 (2007)

25. He, C, Wang, Y: Remark on the regularity for weak solutions to the magnetohydrodynamic equations. Math. Methods Appl. Sci. 31, 1667-1684 (2008)

26. Lei, Z, Zhou, Y: BKM criterion and global weak solutions for magnetohydrodynamics with zero viscosity. Discrete Contin. Dyn. Syst., Ser. A 25, 575-583 (2009)

27. Wang, Y, Zhao, H, Wang, Y: A logarithmally improved blow up criterion of smooth solutions for the three-dimensional MHD equations. Int. J. Math. 23, Article ID 1250027 (2012)

28. $\mathrm{Wu}, \mathrm{J}$ : Viscous and inviscid magneto-hydrodynamics equations. J. Anal. Math. (Jerus.) 73, 251-265 (1997)

29. Zhou, Y: Remarks on regularities for the 3D MHD equations. Discrete Contin. Dyn. Syst. 12, 881-886 (2005)

30. Zhou, Y, Gala, S: Regularity criteria for the solutions to the 3D MHD equations in the multiplier space. Z. Angew. Math Phys. 61, 193-199 (2010)

31. Zhou, Y, Gala, S: A new regularity criterion for weak solutions to the viscous MHD equations in terms of the vorticity field. Nonlinear Anal. 72, 3643-3648 (2010)

32. Zhou, Y, Gala, S: Regularity criteria for the solutions to the 3D MHD equations in the multiplier space. Z. Angew. Math. Phys. 61, 193-199 (2010)

33. Admas, RA: Sobolev Spaces. Academic Press, New York (1975)

34. Galdi, GP: An Introduction to the Mathematical Theory of the Navier-Stokes Equations. Vols I, II. Springer, New York (1994)

35. Ladyzhenskaya, OA: Mathematical Theory of Viscous Incompressible Flow, 2nd edn. Gordon \& Breach, New York (1969). English translation

\section{Submit your manuscript to a SpringerOpen ${ }^{\mathcal{D}}$ journal and benefit from:}

- Convenient online submission

Rigorous peer review

- Immediate publication on acceptance

- Open access: articles freely available online

- High visibility within the field

- Retaining the copyright to your article 